\title{
IGF-dependent dynamic modulation of a protease cleavage site in the intrinsically disordered linker domain of human IGFBP2
}

\author{
Garima Jaipuria ${ }^{1}$, Divya Shet ${ }^{1}$, Shahid Malik ${ }^{1}$, Monalisa Swain ${ }^{1}$, Hanudatta S. Atreya ${ }^{1}$, \\ Charles A. Galea ${ }^{2}$, Mark G. Slomiany ${ }^{3}$, Steven A. Rosenzweig ${ }^{3}$, Briony E. Forbes ${ }^{4}$, \\ Raymond Norton ${ }^{2}$, and Somnath Mondal ${ }^{1}$ \\ ${ }^{1}$ Indian Institute of Science \\ ${ }^{2}$ Monash Institute of Pharmaceutical Sciences \\ ${ }^{3}$ Medical University of South Carolina \\ ${ }^{4}$ Flinders University
}

November 24, 2021

\begin{abstract}
Functional regulation via conformational dynamics is well known in structured proteins, but less well characterized in intrinsically disordered proteins and their complexes. Using NMR spectroscopy we have identified a dynamic regulatory mechanism in the human insulin-like growth factor (IGF) system involving the central, intrinsically disordered linker domain of human IGFbinding protein-2 ( $h$ IGFBP2). The bioavailability of IGFs is regulated by the proteolysis of IGF-binding proteins. In the case of $h$ IGFBP2, the linker domain (L- $h$ IGFBP2) retains its intrinsic disorder upon binding IGF-1 but its dynamics are significantly altered, both in the IGF binding region and distantly located protease cleavage sites. The increase in flexibility of the linker domain upon IGF-1 binding may explain the IGF-dependent modulation of proteolysis of IGFBP2 in this domain. As IGF homeostasis is important for cell growth and function, and its dysregulation is a key contributor to several cancers, our findings open up new avenues for the design of IGFBP analogs inhibiting IGF-dependent tumors.
\end{abstract}

IGF-dependent dynamic modulation of a protease cleavage site in the intrinsically disordered linker domain of human IGFBP2 


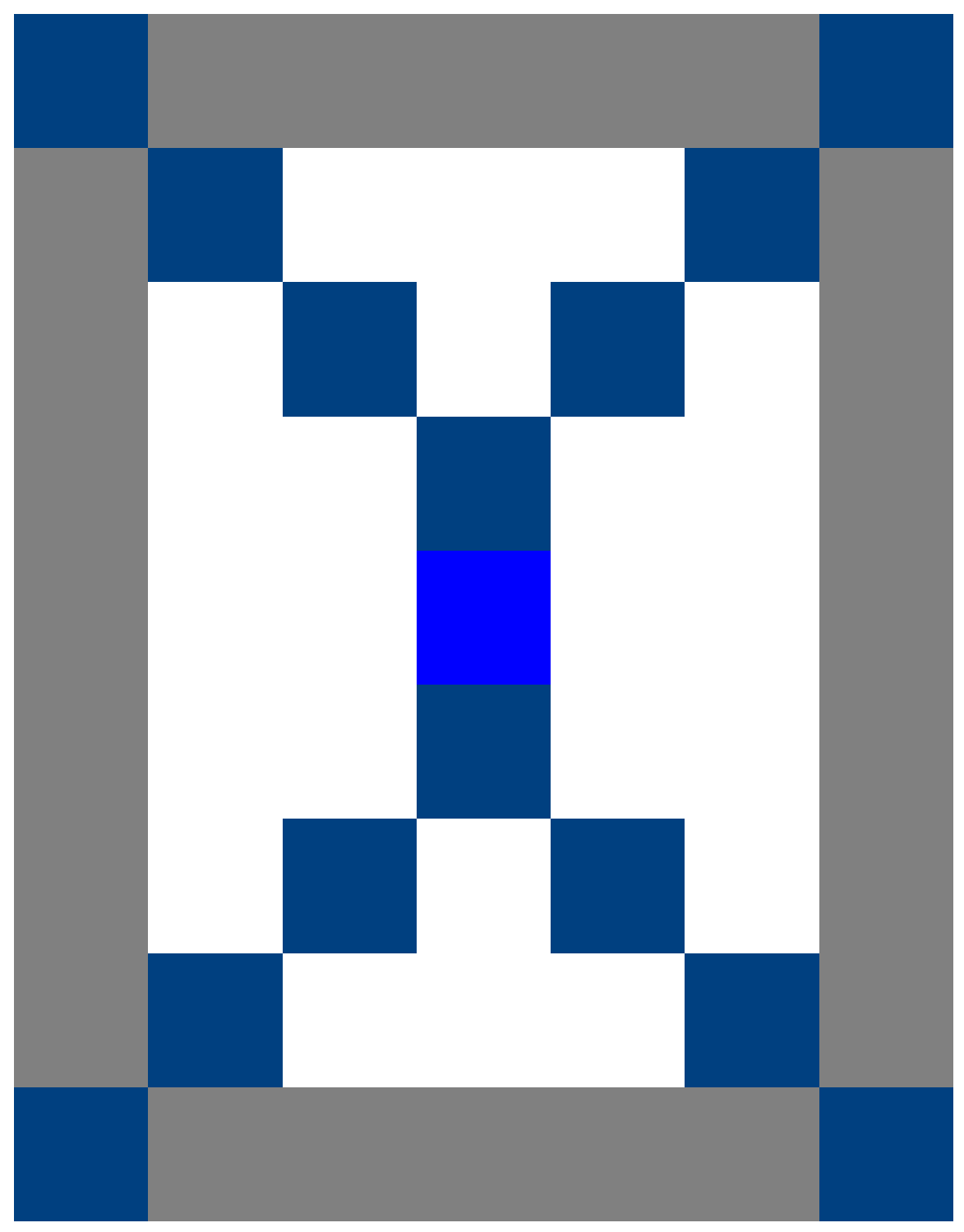

Garima Jaipuria, ${ }^{a^{*}}$

Divya Shet, ${ }^{\mathrm{a}, \mathrm{b}^{*}}$ Shahid Malik, ${ }^{\mathrm{a}}$ Monalisa Swain, ${ }^{\mathrm{a}, \mathrm{c}^{*}}$ Hanudatta S. Atreya, ${ }^{\mathrm{a}}$ 


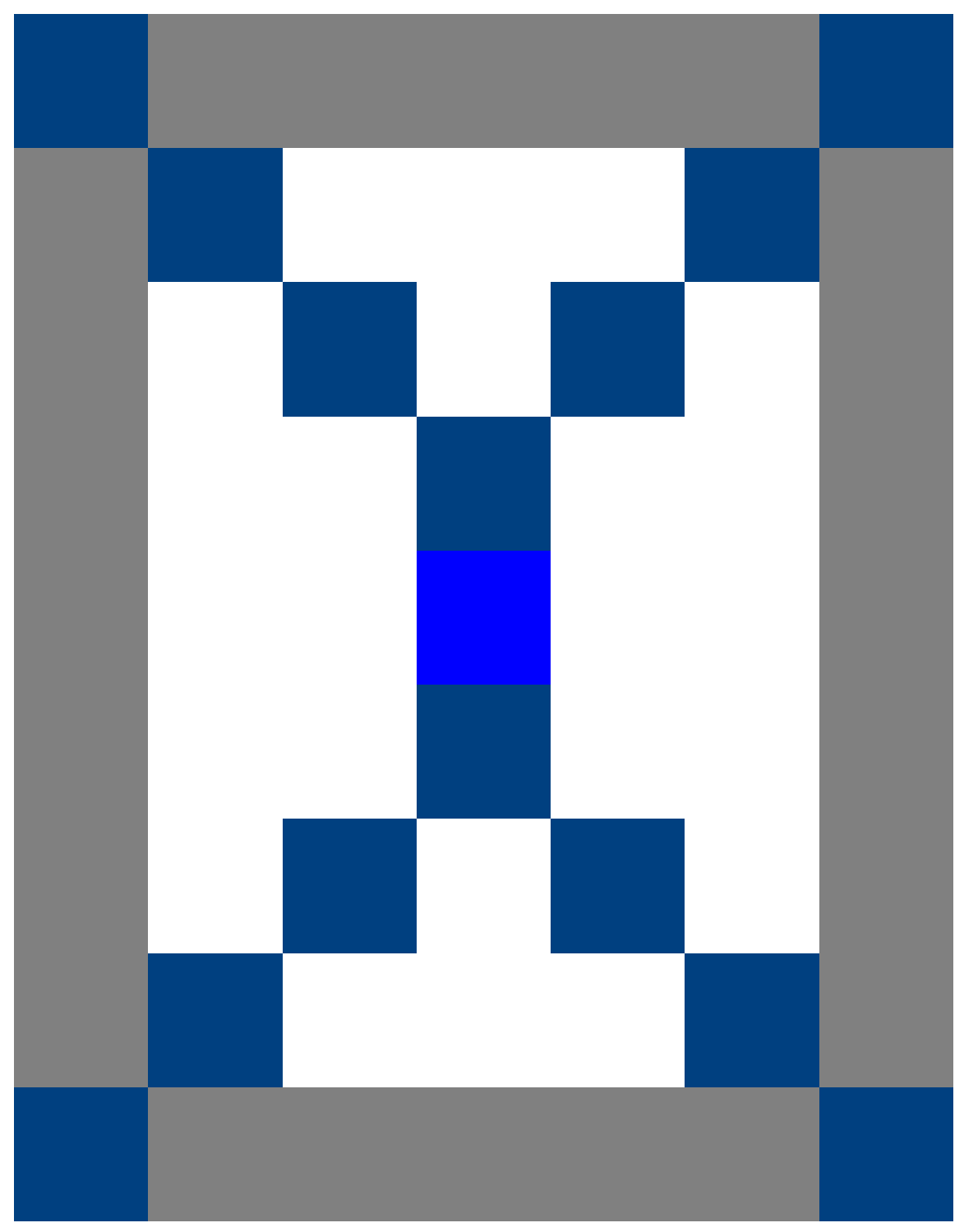

Charles A. Galea, ${ }^{\mathrm{d}}$ Mark G. Slomiany, ${ }^{\mathrm{e}}$ Steven A. Rosenzweig, ${ }^{\mathrm{e} B r i o n y}$ E. Forbes, ${ }^{\mathrm{f}}$ Raymond S. Norton, ${ }^{\mathrm{d}, \mathrm{g}}$ Somnath Mondal ${ }^{\mathrm{a}, \mathrm{h}^{*}}$

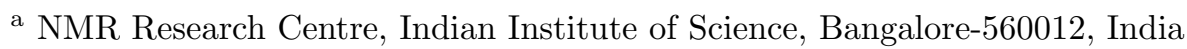

b Nanobiophysics lab, Raman Research Institute, Sadashivnagar, Bangalore-80, India

${ }^{\mathrm{c}}$ Frederick National Laboratory for Cancer Research, Maryland-21701, USA

${ }^{d}$ Medicinal Chemistry, Monash Institute of Pharmaceutical Sciences, Parkville 3052, Australia

e Department of Cell and Molecular Pharmacology, Medical University of South Carolina, Charleston SC 29425, USA

${ }^{\mathrm{f}}$ Flinders Health and Medical Research Institute, Flinders University, SA 5042, Australia

g ARC Centre for Fragment-Based Design, Monash University, Parkville 3052, Australia 


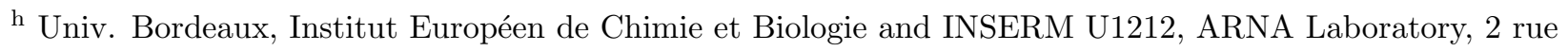
Robert Escarpit, 33607 Pessac Cedex, Bordeaux, France

*These authors contributed equally

Corresponding authors: Garima Jaipuria (garimajaipuria@gmail.com) and Somnath Mondal (somomsom@gmail.com)

\begin{abstract}
Functional regulation via conformational dynamics is well known in structured proteins, but less well characterized in intrinsically disordered proteins and their complexes. Using NMR spectroscopy we have identified a dynamic regulatory mechanism in the human insulin-like growth factor (IGF) system involving the central, intrinsically disordered linker domain of human IGF-binding protein-2 ( $h$ IGFBP2). The bioavailability of IGFs is regulated by the proteolysis of IGF-binding proteins. In the case of $h$ IGFBP2, the linker domain (L- $h$ IGFBP2) retains its intrinsic disorder upon binding IGF-1 but its dynamics are significantly altered, both in the IGF binding region and distantly located protease cleavage sites. The increase in flexibility of the linker domain upon IGF-1 binding may explain the IGF-dependent modulation of proteolysis of IGFBP2 in this domain. As IGF homeostasis is important for cell growth and function, and its dysregulation is a key contributor to several cancers, our findings open up new avenues for the design of IGFBP analogs inhibiting IGF-dependent tumors.
\end{abstract}

\title{
Introduction
}

The insulin-like growth factor (IGF) system plays an essential role in cell growth, differentiation, and function, and in recent years has become an important target for cancer therapeutics, with more than 30 anti-cancer drugs focusing on this system. This system consists of two peptide hormones: IGF-1 and -2; the receptors: IGF-1R and IGF-2R; six soluble, high-affinity IGF-binding proteins (IGFBPs; numbered 1-6) and IGFBP proteases (Fig. 1 ). IGF-1 and -2 are small proteins $(\sim 7.5 \mathrm{kDa})$ that circulate in the bloodstream and function in signaling by binding to IGF receptors. The biological activities of the IGFs are modulated by IGFBPs, which bind IGFs with higher affinities than the IGF-1R, thereby restricting their delivery to the IGF-1R. Proteolysis of the IGFBPs dissociates IGFs from the complex, enabling them to bind to and activate their receptors (Fig. 1 ).

The biological actions of the IGF: IGFBP: IGF-R axis has been studied extensively. However, a comprehensive understanding of the structural basis for IGF-IGFBP interactions is still lacking. The IGFBPs consist of structured globular N- and C-domains joined by a central (linker) domain. The three-dimensional structures of full-length IGFBPs have not yet been determined, although structures are available for the N- and Cterminal domains of the different IGFBPs either free in solution or bound to IGFs. These studies have shown that all IGFBPs interact similarly with IGFs and that binding sites for the IGFs are located primarily in the $\mathrm{N}$-terminal and C-terminal domains of IGFBPs. The intrinsically disordered linker domain, which contains several post-translational modification motifs and the IGFBP-protease cleavage sites, has been proposed to merely tether the N- and C-terminal domains. However, deletion of the linker domain from full-length proteins results in the loss of IGF binding affinity, indicating that this domain may contribute to IGF-binding in the full-length proteins. Further, in some of the IGFBPs, proteolysis in the linker domain requires the binding of IGFs. Despite its apparent importance in IGFBP function, the structure of the linker domain and its interaction with IGFs have not been characterized. Here we have investigated the interaction of the linker domain of human IGFBP2 (L- $h$ IGFBP2; residues A97-C191) with IGFs using NMR spectroscopy and surface plasmon resonance (SPR). Our study confirms that L- $h$ IGFBP2 is intrinsically disordered and shows that it retains moderate binding affinity to IGFs $\left(K_{\mathrm{d}} \sim 4 \mu \mathrm{M}\right)$. IGF binding has a specific effect on the dynamics of L- $h$ IGFBP2 residues that interact with IGF-1, but also on the protease cleavage sites. This altered dynamics may explain the IGF-dependent proteolysis of IGFBP2 in this domain. Our understanding of the role of dynamics in the functional regulation of the IGF-system offers new insights that will help guide the design and development of IGFBP- based analogs for inhibiting IGF-IGF-1R signaling and growth of IGF-dependent tumors. 


\section{Results}

\subsection{NMR Studies of L-hIGFBP2}

L- $h$ IGFBP2 was cloned, overexpressed, and purified as described in Materials and Methods. The purified protein had a molecular mass of $12.2 \mathrm{kDa}$ as verified by MALDI-TOF mass spectrometry (expected: 12.211 $\mathrm{kDa}$ ) and migrated at ${ }^{\sim} 20 \mathrm{kDa}$ on SDS-PAGE (Fig. S1 ). Such aberrant mobility on SDS-PAGE is typical of intrinsically disordered proteins (IDPs) and has been described for the linker domain of IGFBP5. The 2D $\left[{ }^{15} \mathrm{~N}-{ }^{1} \mathrm{H}\right]$ heteronuclear single quantum coherence (HSQC) spectrum of recombinant L- $h$ IGFBP2 (Fig. 2a ) shows a unique ${ }^{15} \mathrm{~N}-{ }^{1} \mathrm{H}$ correlation for each residue, indicating that conformational averaging is fast on the NMR time scale. Comparison of the 2D $\left[{ }^{15} \mathrm{~N}-{ }^{1} \mathrm{H}\right]$ HSQC spectra (Fig. S2 ) for L- $h$ IGFBP2 (in red) and full-length $h$ IGFBP2(in blue) shows mainly overlap of all residues in the linker region regardless of whether they are part of the full-length protein or in isolation, implying that the linker domain is also disordered in the full-length protein.

The limited ${ }^{1} \mathrm{H}$ chemical shift dispersion of the $2 \mathrm{D}\left[{ }^{15} \mathrm{~N}-{ }^{1} \mathrm{H}\right]$ HSQC spectrum implies that L- $h$ IGFBP2 is unstructured. In support of this, ${ }^{3} J$ HNH $\alpha$ coupling constants (measured using G-matrix Fourier transform (GFT) $(3,2)$ D HNHA), a lack of deviations of backbone chemical shifts from random coil values, and secondary structure propensity prediction also indicate an overall lack of ordered secondary structure (Fig. S3 ). The NH bond order parameter estimation based on the chemical shifts of the backbone $\left({ }^{1} \mathrm{H}^{\alpha},{ }^{13} \mathrm{C}^{\alpha}\right.$, $\left.{ }^{13} \mathrm{C}^{\prime},{ }^{15} \mathrm{~N}\right)$ and sidechain $\left({ }^{13} \mathrm{C}^{\beta}\right)$ nuclei confirms a high degree of disorder in L- $h$ IGFBP2 (Fig. 2b ), in agreement with the general consensus that the linker domains of all IGFBPs are disordered (Fig. S4 ). The backbone ${ }^{1} \mathrm{H}$ amide exchange rates could not be obtained from $2 \mathrm{D}^{15} \mathrm{~N}-{ }^{1} \mathrm{H}$ HSQC spectra, as the decrease in intensity of cross-peaks in the 2D spectrum upon dissolving the protein in $100 \%{ }^{2} \mathrm{H}_{2} \mathrm{O}$ was too fast to be measurable. The exchange rates were therefore characterized from $2 \mathrm{D}^{13} \mathrm{CO}-{ }^{15} \mathrm{~N}$ EXSY at $\mathrm{pH} 6$ and $20{ }^{\circ} \mathrm{C}$, which indicated an upper limit of $k_{e x} \sim 1 \mathrm{~s}^{-1}$ for $k$ ex for all residues for a 1:1 mixture of $\mathrm{H}_{2} \mathrm{O}$ and $\mathrm{H}_{2} \mathrm{O}$ (Fig. S5).

\subsection{Binding of ${ }^{15} \mathrm{~N} L-h I G F B P 2$ to $I G F-1$ and $I G F-2$}

Dissociation constants $\left(K_{\mathrm{d}}\right)$ of $4.1 \pm 2.2 \mu \mathrm{M}$ for IGF-1 and $3.7 \pm 1.5 \mu \mathrm{M}$ for IGF-2 were estimated from surface plasmon resonance (SPR) based on the association and dissociation rates (Figs. 3a, b ). The binding of IGF-1 and IGF-2 to L- $h$ IGFBP2 was also monitored using NMR. To identify L- $h$ IGFBP2 residues involved in binding IGFs, a sample of ${ }^{15} \mathrm{~N}$-labeled L- $h$ IGFBP2 was titrated with unlabeled IGF-1. Fig. 3c shows an overlay of the $2 \mathrm{D}\left[{ }^{15} \mathrm{~N}-{ }^{1} \mathrm{H}\right]$ HSQC spectrum of the complex with that of free (unbound) L- $h$ IGFBP2, with residues undergoing significant shifts highlighted in the inset. For $1 \mathrm{mM}$ IGFBP2 protein, the peaks stop moving after the addition of $1 \mathrm{mM}$ IGF-1, showing a clear 1:1 ratio for binding. Therefore, the binding is both very dynamic (fast on and off rates) and of moderate affinity ( $K_{\mathrm{d}}$ of $4.1 \pm 2.2 \mu \mathrm{M}$ as measured from SPR,Fig. 3a ). However, a substantial change in the overall dynamics of the protein was observed, as discussed below. Assignment of the shifted resonances was based on inspection of their position for the unbound L- $h$ IGFBP2 spectrum. The magnitude of ${ }^{15} \mathrm{~N} /{ }^{1} \mathrm{H}$ chemical shift perturbations for each residue of L- $h$ IGFBP2 (Fig. 3d ) shows that residues K150-E161 and Q165 undergo the largest chemical shift changes upon binding IGF-1, with an average shift of $0.032 \pm 0.017 \mathrm{ppm}$. Similar shifts were observed when IGF-1 bound to full-length IGFBP2 (Fig. S6 ). A region containing these residues (K150-E161) is predicted to be a molecular recognition feature (MoRF) (a short binding region located within a longer intrinsically disordered region) and is located adjacent to the protease cleavage sites of $h$ IGFBP2 (Figs. 3e ). To confirm the involvement of these residues in binding IGF-1, we prepared a mutant form of L-IGFBP2 with deletion of K150-E161 (L-hIGFBP2[desK150-E161]), which showed weaker binding to IGF-1 (Fig. S7 ) .

We also identified residues in IGF-1 involved in binding L- $h$ IGFBP2. For this purpose, ${ }^{15}$ N-labeled IGF-1 in $50 \mathrm{mM}$ Na-phosphate buffer ( $\mathrm{pH}$ 6.0) was titrated at $20^{\circ} \mathrm{C}$ with a solution of unlabeled L- $h$ IGFBP2. An overlay of the 2D $\left[{ }^{15} \mathrm{~N}-{ }^{1} \mathrm{H}\right]$ HSQC spectrum of the L- $h$ IGFBP2: IGF-1 complex with that of free IGF-1 is shown inFig. S8. Chemical shifts for most residues of IGF-1 remained unchanged, but cross-peaks for 
residues E3, T4, A13, V17, C18, G19, D20, R21, G22, M59, Y60, C61, and A62, which were very weak or absent in free IGF-1, exhibited a significant increase in intensity in the bound form (Fig. S8). This implies that the dynamics of IGF- 1 are also significantly affected upon binding L- $h$ IGFBP2, even though its average conformation is unchanged (Fig. S8).

\subsection{Backbone Dynamics of L-hIGFBP2 from ${ }^{15} \mathrm{~N}$ relaxation}

Insight into the functional regulation of IGF-1 by L- $h$ IGFBP2 was achieved by studying the dynamics of L- $h$ IGFBP2 in the free and IGF-1-bound forms using ${ }^{15} \mathrm{~N}$ relaxation. Reduced spectral density mapping was used to examine dynamics in the microsecond-millisecond ( $\mu \mathrm{s}-\mathrm{ms})$ and picosecond-nanosecond (ps-ns) regimes. The ${ }^{15} \mathrm{~N}$ relaxation rates $\left(\mathrm{R}_{1}, \mathrm{R}_{2}, \mathrm{R}_{1 \rho}\right)$ and ${ }^{15} \mathrm{~N}^{-1} \mathrm{H}$ heteronuclear nuclear Overhauser effects (HetNOE) were measured at a ${ }^{1} \mathrm{H}$ resonance frequency of $800 \mathrm{MHz}$ (Fig. 4 ). Based on ${ }^{15} \mathrm{~N} \mathrm{R}_{1}$ and $\mathrm{R}_{1}$ relaxation values, an average overall rotational correlation time of $\sim 3$ ns for the disordered linker domain was obtained for unbound L- $h$ IGFBP2. The average overall correlation time for the linker domain in full-length $h$ IGFBP2 $(32 \mathrm{kDa})$ determined by a similar method was $\sim 4 \mathrm{~ns}$ at $293 \mathrm{~K}$, implying that the disordered linker domain retains a high degree of flexibility in the full-length form, largely unaffected by the presence of the N- and C-domains.

The ${ }^{15} \mathrm{~N} \mathrm{R}_{1}, \mathrm{R}_{2}$, and ${ }^{15} \mathrm{~N}-{ }^{1} \mathrm{H}$ het-NOE values and a plot of the calculated spectral density functions- $J(0), J$ $\left(\omega_{\mathrm{N}}\right)$, and $J\left(0.87^{*} \omega_{\mathrm{H}}\right)$ for L- $h$ IGFBP2 in free and IGF-1-bound forms are shown in Fig. 4 and Fig. 5 , respectively. Several important observations can be made. First, L- $h$ IGFBP2 exhibits a high degree of flexibility in both the free and IGF-1-bound forms, as reflected by the $J\left(0.87^{*} \omega_{\mathrm{H}}\right)$ and $J(0)$ values, with the latter being significantly less than $2 / 5 \tau_{c}$ for most residues (where $\tau_{c}$ is the rotational correlation time of a rigid isotropically tumbling protein of equivalent size). Second, the ${ }^{15} \mathrm{~N}$ relaxation rates for residues K150-E161, Q165, and M166 of the linker domain are significantly perturbed by IGF-1 in complex with L- $h$ IGFBP2, as the large complex causes a great increase in correlation time $\left(\tau_{\mathbf{c}}\right)$ which in turn causes the fast $\mathrm{T}_{2}$ relaxation (Fig. 4) . In the full-length $h$ IGFBP2 complex the intensities of cross-peaks corresponding to these residues in the 2D $\left[{ }^{15} \mathrm{~N},{ }^{1} \mathrm{H}\right]$ HSQC spectrum are reduced owing to the formation of a large complex which causes an increase in correlation time $\left(\tau_{\mathbf{c}}\right)$, resulting in the fast $\mathrm{T}_{2}$ relaxation and increased NMR line-width (Fig. S6 ). Third, large $J(0)$ values indicative of dynamics in the $\mu$ s-ms regime are significantly enhanced for L-domain residues involved in binding IGF-1 (K150-E161), as well as those distant from the binding site (V110, N113, H117, H172, Q165, M166, L174, and L182). This increase in $J(0)$ values can be attributed to the larger size of the complex and slow conformational exchange in the $\mu$ s-ms regime, and is quantified by the exchange rate, $R_{\text {ex }}$, which was estimated by measuring the ${ }^{15} \mathrm{~N}$ transverse relaxation rate in the rotating frame $\left(\mathrm{R}_{1 \rho}\right)$ at $800 \mathrm{MHz}$ for both the unbound and bound forms of L- $h$ IGFBP2. Values of $\mathrm{R}_{\text {ex }}$ calculated using the difference in $J(0)$ values obtained with $\mathrm{R}_{2}$ and $\mathrm{R}_{1 \rho}$ are plotted in Fig. 5 . Notably, an overall increase in $R_{\text {ex }}$ is observed in the IGF-bound complex for residues of L- $h$ IGFBP2 both close to and distant from the binding site. This implies that in the IGF-1-bound complex L- $h$ IGFBP2 populates an ensemble of alternate conformations that interconvert on the $\mu \mathrm{s}-\mathrm{ms}$ timescale. The region bound to IGF-1 exhibits a 'reduced' level of conformational dynamics, such that some of the ns-ps timescales have now entered the $\mu \mathrm{s}-\mathrm{ms}$ timescale (and therefore now entered the exchange regime in these experiments). Interestingly, it is known that proteolytic cleavage of $h$ IGFBP2 by the pregnancy-associated plasma protein-A (PAPP-A) is enhanced in the IGF-1-bound state. As shown in Fig. 7, the region K150-E161 from the disordered linker domain of $h$ IGFBP2 has a helical propensity, as predicted by AlphaFold; this binds to IGF-1 and shows a change in exchange in $\mu \mathrm{s}-\mathrm{ms}$ timescale due to the formation of the larger complex upon binding. The IGF-1 bound complex has a higher correlation time $\left(\tau_{\mathbf{c}}\right)$ which in turn causes the fast $\mathrm{T}_{2}$ relaxation, resulting in a higher relaxation rate, as the relaxation times and relaxation rates are simple inverses of each other. The majority of the L- $h$ IGFBP2 residues recognized by proteases (Fig. 3 ) show increases in $R_{\text {ex }}$ upon IGF binding (Fig. 5 ). This explains the IGF-dependent dynamic modulation of a protease cleavage site region in the intrinsically disordered linker domain of $h$ IGFBP2.

To estimate the conformational entropy associated with binding IGF-1, approximate backbone NH order parameters $\left(S^{2}\right)$ using $J(0)$ and $J\left(\omega_{\mathrm{N}}\right)$ were calculated for L- $h$ IGFBP2. $J(0)$ and $J\left(\omega_{\mathrm{N}}\right)$ calculated from 
$\mathrm{R}_{1 \rho}$ were used to avoid the effect of conformational exchange when estimating $S^{2}$ values. Order parameters were calculated for both free and bound forms of L- $h$ IGFBP2 and the change in conformational entropy $(\Delta \mathrm{S})$ was estimated using the calculated $S^{2}$ values (for residues with $S^{2}<1$ ) (Eq. 2; Fig. 6 ). The overall $\Delta \mathrm{S}$ value (summed over all residues) of $\sim 100 \mathrm{~J} / \mathrm{mole}(0.024 \mathrm{kcal} / \mathrm{mol})$ implies an increase in entropy for the system upon IGF-1 binding. The contribution of the conformational entropy to the free energy of binding is given by $-\mathrm{T} \Delta \mathrm{S}$, which yields a contribution of $-7 \mathrm{kcal} / \mathrm{mol}$.

\section{Discussion}

In recent years, the concept of "fuzzy complexes" in IDPs has been described, which proposes that functionally important regions of IDPs in protein complexes can retain their structural disorder. In fuzzy complexes, dynamic regulation ensues when the ensemble average population of conformers of the IDP and/or their flexibility are affected upon ligand binding. The current study involving the intrinsically disordered linker domain of human IGFBP2 exemplifies such a case.

In the IGF system, proteolysis plays a crucial role in regulating the bioavailability of IGFs. IGFBP levels are regulated by proteolysis following their secretion from the cell and the resulting proteolytic fragments have reduced affinity for IGF ligands. The net effect is an increase in IGFs availability for interaction with the IGF-1R. Thus, efforts to reduce protease action could have a beneficial effect on reducing IGF-1R activity in cancer. Proteolysis of IGFBPs has been observed to be both IGF-dependent and IGF-independent; IGFdependent proteolysis has been observed for IGFBP2 and in IGFBP4, whereas IGFBP3 and IGFBP5 undergo IGF-independent proteolysis. K150-E161 residues in IGFBP2 are more ordered (less disorder disposition, Fig. S4 ), and our experimental results show an increase in dynamics for those residues in IGFBP2 after binding with IGF-1. As these K150-E161 residues in the linker region become more flexible after binding, the conformational ensemble populated by the linker domain of IGFBP2 shifts so that it is more readily recognized by the protease and/or is more amenable to proteolysis. This brings an interesting link for why binding to IGF-1 can be so important for proteolysis for $h$ IGFBP2, which is not the case for IGFBP3 and IGFBP5. An example of an IGF-dependent protease action on $h$ IGFBP2 is PAPP- A, which cleaves $h$ IGFBP2 in an IGF-dependent manner at a single site between Gln165 and Met166, to yield two proteolytic fragments having weak IGF binding affinity.

The different susceptibilities of the different IGFBPs to proteolysis have been attributed to ligand-induced conformational changes. Our studies demonstrate that K150-E161, Q165, and M166 residues of L- $h$ IGFBP2, which are involved in binding IGF-1, exhibit enhanced conformational exchange upon IGF binding (Fig. 4 ). Moreover, this enhanced conformational exchange is not confined to the binding site but extends to some distant residues (V110, N113, H117, H172, Q165, M166, L174, and L182) (Fig. 4 ) and is accompanied by an increase in conformational entropy. This implies distant dynamic regulation, where changes in protein dynamics induced by ligand binding extend to residues distant from the ligand-binding site, even in the absence of a well-defined conformational change. The increase in $\mu s-m s$ motions of residues in the vicinity of protease cleavage sites of L- $h$ IGFBP 2 provides an interesting link to proteolytic cleavage upon binding IGF-1 as it is well known that changes in conformational dynamics upon ligand binding are important for regulation of proteolysis. In the presence of IGF-1, the conformational ensemble populated by the linker domain of IGFBP2 shifts so that it is more readily recognized by the protease and/or is more amenable to proteolysis. Considering the current findings, we therefore propose that dynamic regulation in the linker domain of IGFBP2 plays an important role in its susceptibility to PAPP-A proteolytic cleavage.

These results have significant implications for the development of IGFBPs (mutants and/or chimeras) as antagonists of IGF-1R activation that can block IGF-1R mediated tumor progression. Most current cancer therapeutics target the IGF-signaling pathway and focus on blocking the IGF-1R directly (kinase inhibitors) and/or its downstream effectors. However, a drawback of this approach is the resulting high serum IGF-1 levels in response to targeted inhibition of IGF-1R and adverse side-effects and/or toxicities arising from potential interference with the insulin pathway. It has been suggested recently that therapeutics targeting the interaction of IGFs with IGFBPs may overcome these serious drawbacks. For example, IGFBPs engineered to be protease-resistant by mutating or deleting the protease cleavage sites in the linker domain should act 
as IGF antagonists. Recently, in separate studies, engineered protease-resistant $h$ IGFBP2 and $h$ IGFBP4 were found to inhibit tumor growth in breast cancer. Interestingly, the engineered protease-resistant form of $h$ IGFBP2 lacking residues 114-170 (des(114-170)) retains high-affinity binding to IGF-1 and IGF-2, with only a 1.6-2-fold reduction in affinity compared to full-length $h$ IGFBP2. The present study may now explain the loss in binding affinity of des(114-170) towards both IGFs compared to the full-length protein by the fact that residues K150-E161 of the linker domain, which facilitate IGF binding (Fig. S7 ), were deleted from the construct. This suggests that, in addition to alteration of the protease cleavage sites, more potent IGFBP-based antagonists could be designed by considering the binding affinity of the linker domain for the IGFs and taking into account the resulting change in dynamics upon binding. These studies will facilitate the development of future IGFBP-based antagonists.

In summary, our studies of the intrinsically disordered linker domain of human IGFBP2 provide new insights into the regulatory mechanisms in the IGF system. Contrary to currently held models, the intrinsically disordered linker domain of IGFBP2 is involved in binding IGF-1. L- $h$ IGFBP2 does not undergo a welldefined conformational change upon binding its ligand, but binding is accompanied by a significant change in dynamics on both the millisecond-microsecond and picosecond-nanosecond time scales. This is an example of functional regulation in an intrinsically disordered protein complex by dynamic regulation, which is being recognized increasingly in recent years.

\section{Materials and Methods}

\subsection{Cloning, expression, and purification of L-hIGFBP2}

The primers were designed for L- $h$ IGFBP2 [residues $97-191$ of the full-length protein] and its L- $h$ IGFBP mutants (L- $h$ IGFBP2[desK150-E161]) lacking the C-terminal tag and residues 150-161. Oligonucleotide strands 5' CAT GGT ACC GAT GAT GAT GAT AAA AAG CGC CGG GAC GCC GAG TAT G 3' with enterokinase cleavage site and 5' GAC GAA TTC TTA GGG AGT CCT GGC AGG GGG TGG TCG CA 3 ' were used as forward and reverse primers for L- $h$ IGFBP2[desK150-E161]. The insert was cloned between Kpn1 and Xho1 restriction sites of the pET 32a vector having a thioredoxin tag fused to the protein.

The oligonucleotide strands 5' ATTG GGA TCC GAG AAG CGC CGG 3' and 3' ATTG GAA TTCTTA CAG GGA GTC CTG 5' with BamH1 and EcoR1 restriction sites on forward and reverse primers, respectively, were synthesized by MWG-Eurofins and used to amplify L- $h$ IGFBP2 at $65{ }^{\circ} \mathrm{C}$ in a thermo-cycler. Following amplification, PCR products were purified using a Sigma Gel elution kit after analyzing on $1 \%$ agarose gel in 1X TAE buffer. The vector used was an IPTG-inducible pGEX6P-1 with a GST tag. The vector and L- $h$ IGFBP2 were digested with BamH1 and EcoR1, producing staggered ends, and treated with ligase at $16^{\circ} \mathrm{C}$ for $16 \mathrm{~h}$. The ligated product was used to transform $E$. coli Top10 cells, and the desired clone was obtained using a Sigma miniprep plasmid isolation kit. The clone was confirmed by sequencing. During the process of cloning the following C-terminal tag was introduced: KNSRVDSSGRIVTD. This tag did not affect the binding of IGF-1 as verified using the construct L- $h$ IGFBP2[desK150-E161], which did not contain these additional residues. At the N-terminal end, cleavage by HRV 3C protease to separate the GST tag from the protein resulted in the following residues added to the N-terminus: GPLGS.

The L- $h$ IGFBP2 construct was transformed into $E$. coliBL21(DE3) competent cells. The transformed colony was inoculated in $50 \mathrm{~mL}$ of primary culture (LB broth with $100 \mu \mathrm{g} / \mathrm{mL}$ ampicillin) and incubated at $37^{\circ} \mathrm{C}$ for $16 \mathrm{~h}, 200 \mathrm{rpm}$. The cells were transferred to minimal media and grown to the mid-log phase (optical density at $\left.600 \mathrm{~nm}\left(\mathrm{OD}_{600}\right) \sim 0.6\right)$. At this OD, the expression of L- $h$ IGFBP2 was induced with $1 \mathrm{mM}$ isopropyl $\beta$-D-1-thiogalactopyranoside (IPTG) for $5 \mathrm{~h}$ at $37{ }^{\circ} \mathrm{C}, 200 \mathrm{rpm}$.

Cells were harvested by centrifugation at $6000 \mathrm{rpm}$ and resuspended in phosphate-buffered saline (PBS) [150 $\left.\mathrm{mM} \mathrm{NaCl}, 2.5 \mathrm{mM} \mathrm{KCl}, 10 \mathrm{mM} \mathrm{Na}_{2} \mathrm{HPO}_{4}, 2 \mathrm{mM} \mathrm{KH}_{2} \mathrm{PO}_{4}, \mathrm{pH} 7.4\right]$, and $1 \mathrm{mM}$ PMSF. Lysis by sonication was carried out on the ice for $10 \mathrm{~min}(30 \mathrm{~s}$ pulse) and the lysate was centrifuged at $8000 \mathrm{rpm}$ for $40 \mathrm{~min}$. The supernatant was collected and allowed to bind to the pre-equilibrated GST bind resin for $2 \mathrm{~h}$ at $4^{\circ} \mathrm{C}$ on a rotator. The resin was washed three times with each of 10 bed volume of PBS buffer, high salt $25 \mathrm{mM}$ HEPES, $0.05 \% \mathrm{NaN}_{3}, 0.5 \mathrm{M} \mathrm{NaCl}, 0.1 \%$ Triton X-100, pH 7.5] and low salt [25 mM HEPES, $0.5 \% \mathrm{NaN}_{3}, 0.1$ 
$\mathrm{M} \mathrm{NaCl}, 0.1 \%$ TritonX-100, $\mathrm{pH} 7.5]$ buffer. The cleavage buffer (PBS) was used to wash the beads before adding HRV $3 \mathrm{C}$ protease to perform on-column cleavage at $4^{\circ} \mathrm{C}$ for $16 \mathrm{~h}$.

L- $h$ IGFBP2[desK150-E161] was expressed in E. coli BL21 cells at $30{ }^{\circ} \mathrm{C}$ and induced for $4 \mathrm{~h}$ with $0.5 \mathrm{mM}$ IPTG. For the purification of thioredoxin fused L- $h$ IGFBP2[desK150-E161], the cell pellet was resuspended in buffer A (20 mM Tris pH 8.0, $100 \mathrm{mM} \mathrm{NaCl}, 10 \mathrm{mM}$ imidazole, and $10 \%$ glycerol) containing $0.3 \mathrm{mg} / \mathrm{mL}$ lysozyme and incubated for $20 \mathrm{~min}$ in the presence of EDTA-free protease inhibitor cocktail from Roche. The cell resuspension was sonicated, and the cell lysate was clarified by centrifuging at 14,000 rpm for 30 min at $4{ }^{\circ} \mathrm{C}$. The supernatant was loaded onto a pre-equilibrated Ni-NTA HisTrap column (GE Healthcare, $5 \mathrm{ml}$ ) with a flow rate of $0.25 \mathrm{~mL} / \mathrm{min}$. Unbound proteins were removed by extensive washing with buffer A followed by a wash with buffer A containing $0.5 \mathrm{M} \mathrm{NaCl}$. The fusion protein was eluted with buffer A containing $250 \mathrm{mM}$ imidazole and exchanged with enterokinase cleavage buffer (50 mM Tris-Cl, $\mathrm{pH} 8.0,1 \mathrm{mM}$ $\mathrm{CaCl}_{2}$, and $50 \mathrm{mM} \mathrm{NaCl}$ ). The thioredoxin tag was removed by incubating the fusion protein in enterokinase at $23{ }^{\circ} \mathrm{C}$ for $16 \mathrm{~h}$ and passing the mixture back onto the Ni-NTA HisTrap column (GE Healthcare, 5ml). Unbound protein was eluted in buffer A. The protein was concentrated using a Millipore centricon with a 3 $\mathrm{kDa}$ molecular weight cut-off.

\subsection{Expression and purification of full-length hIGFBP2}

A ${ }^{15}$ N-labeled sample of full-length $h$ IGFBP2 was prepared as described previously. Briefly, the $h$ IGFBP2 construct was transformed into E. coli BL21(DE3) Star ${ }^{\mathrm{TM}}$ competent cells. The transformed colony was inoculated in $10 \mathrm{~mL}$ primary culture of LB medium containing $200 \mu \mathrm{g} / \mathrm{ml}$ ampicillin and incubated at $37^{\circ} \mathrm{C}$ for $16 \mathrm{~h}, 200 \mathrm{rpm}$. The cells were then diluted 100-fold into fresh LB (amp) and grown up to a cell density corresponding to $\mathrm{OD}_{600} \sim 0.6$. The cells were centrifuged and the cell pellet transferred to $1 \mathrm{~L}$ minimal media followed by growth at $37^{\circ} \mathrm{C}$ up to a cell density corresponding to $\mathrm{OD}_{600} \sim 0.8$ before inducing the protein expression with $0.5 \mathrm{mM}$ IPTG at $25^{\circ} \mathrm{C}$ for $6 \mathrm{~h}$. Cells were harvested by centrifugation, solubilized in Buffer-I [Phosphate-buffer ( $50 \mathrm{mM}$ sodium phosphate, $0.3 \mathrm{M} \mathrm{NaCl}, \mathrm{pH} 8.0$ ) containing $8 \mathrm{M}$ urea, $5 \mathrm{mM}$ DTT, and $1 \mathrm{mM}$ PMSF] and then sonicated on ice. The supernatant was incubated at $4{ }^{\circ} \mathrm{C}$ for $2 \mathrm{~h}$ with $1 \mathrm{~mL}$ of pre-equilibrated His-Select Nickel affinity agarose (Sigma-Aldrich) followed by washing and elution of the protein with $200 \mathrm{mM}$ imidazole in Buffer-I. The eluate was dialyzed in Buffer-I to remove urea, followed by removal of DTT. The dialyzed protein was exchanged with phosphate buffer ( $50 \mathrm{mM}$ sodium phosphate, 50 $\mathrm{mM} \mathrm{NaCl}, \mathrm{pH} 6.0$ ) and concentrated to $500 \mu \mathrm{L}$ for NMR experiments.

\subsection{SPR studies of L-hIGFBP2 and its mutants}

SPR studies to estimate the binding affinity of IGF-1 and IGF-2 to L- $h$ IGFBP2 and its mutant (lacking the residues 150-161) were carried out on a Biacore 3000 instrument. IGF-1 $(10 \mu \mathrm{g} / \mu \mathrm{L})$ in $10 \mathrm{mM}$ sodium acetate buffer, $\mathrm{pH} 4.0$, was immobilized at a flow rate of $2 \mu \mathrm{L} / \mathrm{min}$ onto the activated CM-5 sensor chip using the amine coupling kit. 520 response units (RU) of IGF-1 were coupled on separate flow cells. The immobilization of pure IGF-2 $(5.5 \mu \mathrm{g} / \mu \mathrm{L})$ was carried out on a separate chip at $25{ }^{\circ} \mathrm{C}$ at a flow rate of 2 $\mu \mathrm{L} / \mathrm{min}$ and 350 response units of IGF-2 were coupled on the flow cell. Binding experiments were carried out for different concentrations of L- $h$ IGFBP2 and its mutants in HBS buffer $(10 \mathrm{mM}$ HEPES, $150 \mathrm{mM} \mathrm{NaCl}$, $3 \mathrm{mM}$ EDTA, $0.005 \%$ surfactant $\mathrm{P} 20, \mathrm{pH}$ 7.5) at a flow rate of $10 \mathrm{uL} / \mathrm{min}$. Regeneration of the biosensor surface before the next reading was done by passing $5 \mu \mathrm{L}$ of $2 \mathrm{M} \mathrm{MgCl}_{2}$ across the chip. The data were fit to the Langmuir 1:1 binding model using the fitting procedures in BIA-evaluation software $3 \cdot 0 \cdot 1$ to calculate dissociation constants.

\subsection{Sequence-specific resonance assignments}

NMR spectra were acquired at $20{ }^{\circ} \mathrm{C}$ on an $800 \mathrm{MHz}$ NMR spectrometer equipped with a triple-resonance cryogenic probe. The following spectra (with measurement times) were acquired for sequence-specific resonance assignment of backbone and side-chain nuclei: 2D HSQC $(0.6 \mathrm{~h}), 3 \mathrm{D}$ HNCO $(4.2 \mathrm{~h}), 3 \mathrm{D}$ HNCACB $(13$ h), 3D CBCACONH (13 h), 3D HBHACONH (6.8 h), 3D (H)C(CO)NH TOCSY (10.4 h), 3D ${ }^{13} \mathrm{C}-\mathrm{TOCSY}-$ Methyl COSY (11.2 h), Reduced Dimensionality (RD) 3D HN(CA)NH (12.4 h), GFT (3,2)D HNHA (6 h). Data were processed with NMRPipe and analyzed using XEASY. Resonance assignments were obtained 
using a semi-automated approach in the program AUTOASSIGN. Sequence-specific resonance assignments were aided by amino acid selective unlabeling of Arg, Asn, Thr, Ser, Gly, and Ala and reduced dimensionality. The complete chemical shifts assignments have been deposited in the BioMagResBank (accession code: 19475).

\subsection{Backbone Dynamics of L-hIGFBP2 from ${ }^{15} \mathrm{~N}$ relaxation}

The experiments for dynamics studies of ${ }^{15} \mathrm{~N}$-labeled hL-IGFBP2 in the presence and absence of IGF-1 were performed at $20{ }^{\circ} \mathrm{C}$ on a Bruker $800 \mathrm{MHz}$ NMR spectrometer equipped with a cryogenic probe. For $\mathrm{R}_{1}$ measurements, eight different time points were collected with relaxation delay periods of $0.01 \mathrm{~s}, 0.05 \mathrm{~s}, 0.1$ $\mathrm{s}, 0.2 \mathrm{~s}, 0.4 \mathrm{~s}, 0.8 \mathrm{~s}, 1 \mathrm{~s}$, and $1.5 \mathrm{~s} . \mathrm{R}_{2}$ and $\mathrm{R}_{1 \rho}$ measurements were carried out at eight different time points with relaxation delay periods of $0.01 \mathrm{~s}, 0.03 \mathrm{~s}, 0.05 \mathrm{~s}, 0.1 \mathrm{~s}, 0.13 \mathrm{~s}, 0.17 \mathrm{~s}, 0.2 \mathrm{~s}, 0.23 \mathrm{~s}$. For the $\mathrm{R}_{1}, \mathrm{R}_{2}, \mathrm{R}_{1 \rho}$ experiments: 2 scans with an interscan relaxation delay of $2.5 \mathrm{~s}, 256$ points, and a spectral window of 24 ppm (65 ms acquisition time) were used in the ${ }^{15} \mathrm{~N}$ dimension. The ${ }^{1} \mathrm{H}$ dimension was acquired using 2048 points $\left(t_{\max }=106 \mathrm{~ms}\right)$ over a 12 -ppm spectral width. For $\mathrm{R}_{1_{\rho}}$ a $4 \mathrm{~ms}$ spin-lock block with a field strength of $2 \mathrm{kHz}$ was used. The same data sets with identical time points, relaxation delays, and experimental time were recorded for the IGF-1-bound form of L- $h$ IGFBP2. All the relaxation experiments were processed with a shifted sine-bell function and zero-filled with twice the time domain points in both dimensions. $\left[{ }^{15} \mathrm{~N}-{ }^{1} \mathrm{H}\right]$ Het-NOE spectra for the free and bound form of L- $h$ IGFBP2 were recorded with 32 transients for $23 \mathrm{~h}$ each with 2048 and 256 points in the direct and indirect dimensions, respectively. An interscan delay of $5 \mathrm{~s}$ was used and acquisition times were $80 \mathrm{~ms}$ in the direct and $65 \mathrm{~ms}$ in the indirect dimension.

An approximate $\mathrm{S}^{2}$ to estimate the conformational entropy of the $\mathrm{NH}$ bond vector was calculated using Equation 1 and the entropy was calculated using Equation 2. 


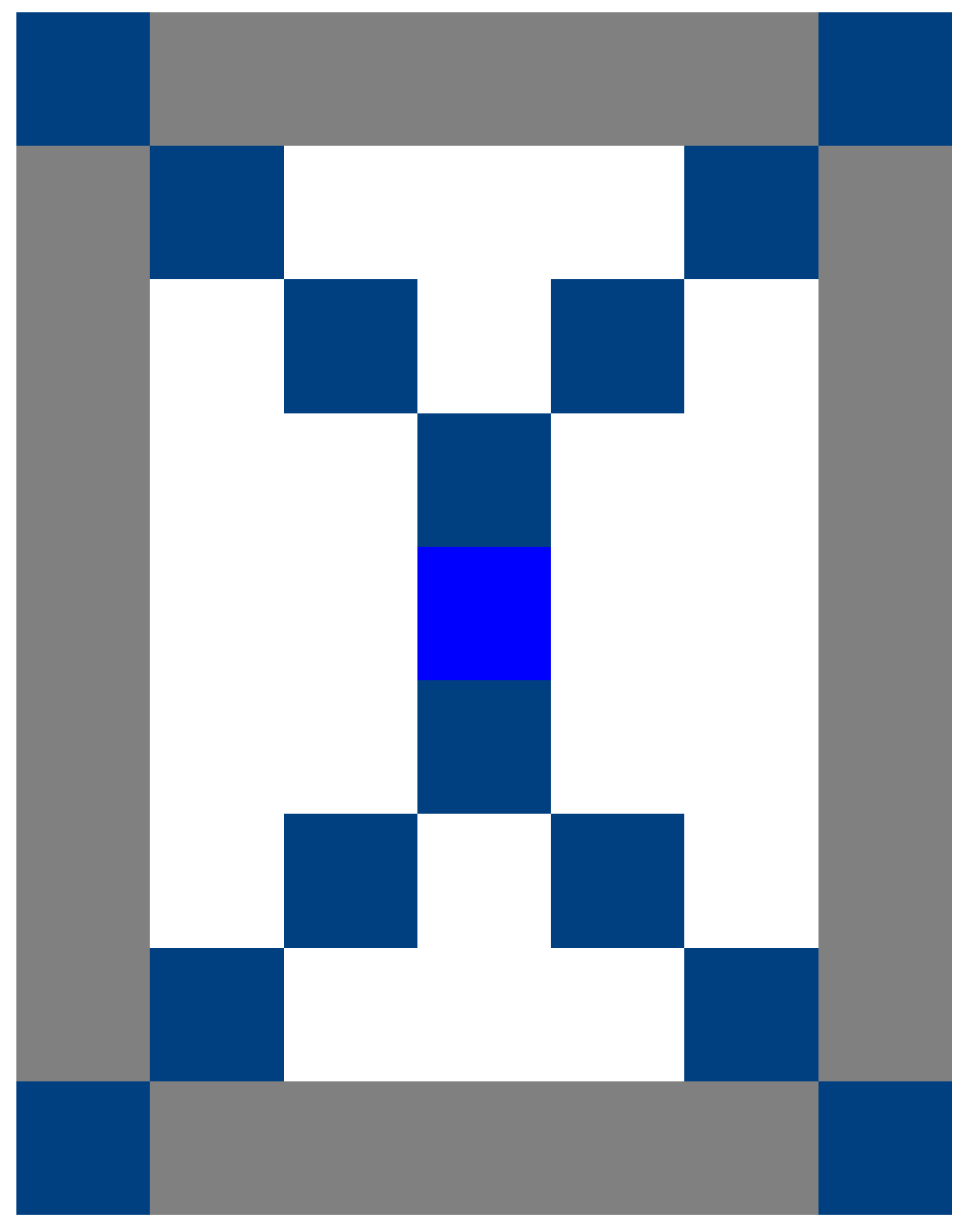




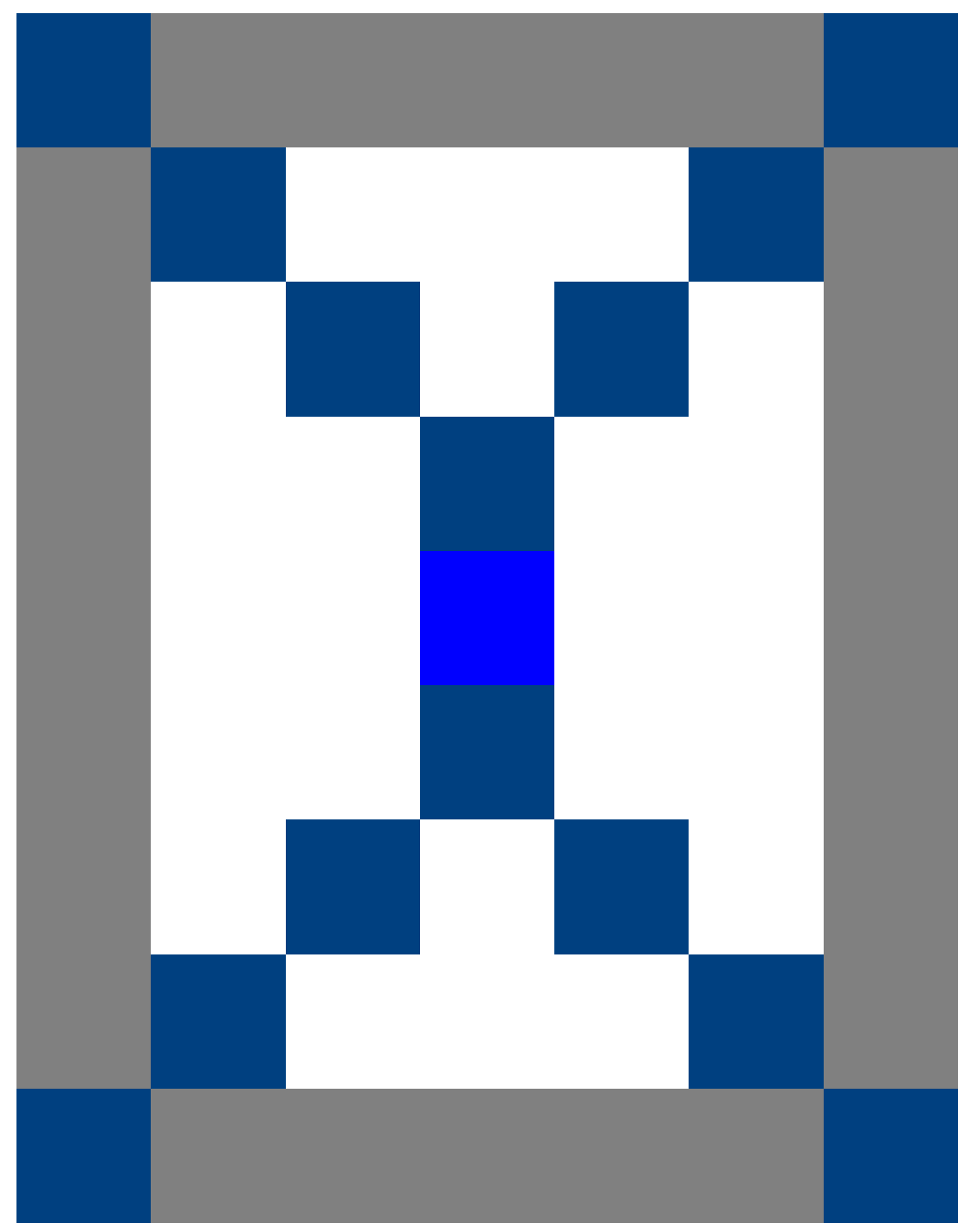

The entropy estimated in this manner is considered as an upper limit due to the inherent assumption that the $\mathrm{NH}$ bond fluctuations for each residue are independent of others.

\section{Acknowledgments}

The NMR Research Centre supported by the Department of Science and Technology (DST), India, and the SPR facility at IISc are gratefully acknowledged. HSA acknowledges research grant support from DAEBRNS. RSN acknowledges fellowship support by the National Health and Medical Research Council of Australia. SAR was supported by the NIH grant CA138313. GJ acknowledges support from IISc and CSIR. We acknowledge the assistance provided by Ms. Kerrie McNeil (University of Adelaide, Australia) in the preparation of labeled IGF-1, and thank Dr. Cameron Mackereth (IECB, Bordeaux, France) for his helpful suggestions to improve the quality of the manuscript.

\section{References}

\section{Figure Legends}


Fig. 1 Schematic of the components in the insulin-like growth factor system (top box) and their functional regulation (lower right diagram). IGFBPs tightly bind and sequester IGFs in the serum and inhibit their interaction with the IGF receptor (IGF-1R). IGFBP protease cleavage within the disordered linker domain of the IGFBP releases IGF allowing it to bind to the IGF-R, stimulating intracellular IGF signaling. The crystal structure of the N- and C-domains of IGFBP4 (N-BP-4 (green) and C-BP-4 (blue), respectively) bound to IGF-1 (PDB ID: 2DSR, red) is shown, with the intrinsically disordered linker domain (L-domain) drawn as a dotted line for illustration. In the case of IGFBP2, the N-, C- and the L-domains are each approximately 100 residues in length.

Fig. 2 : (a) Two dimensional $\left[{ }^{15} \mathrm{~N}-{ }^{1} \mathrm{H}\right]$ HSQC NMR spectrum of L- $h$ IGFBP2 acquired at ${ }^{1} \mathrm{H}$ resonance frequency of $800 \mathrm{MHz}$ at $20^{\circ} \mathrm{C}$ in $50 \mathrm{mM} \mathrm{Na-phosphate} \mathrm{buffer} \mathrm{(pH} \mathrm{6.0)} \mathrm{and} 100 \mathrm{mM} \mathrm{NaCl}$. The peaks are numbered according to full-length protein (i.e., starting from 97 to 191), the peaks numbered -5 to -1 are a part of the $\mathrm{N}$ terminal tag while peak numbers 1 to14 belong to the additional C-terminal residues. (b) The predicted order parameter of L- $h$ IGFBP2 as calculated with TALOS+ using the observed ${ }^{1} \mathrm{H},{ }^{15} \mathrm{~N},{ }^{13} \mathrm{C}^{\alpha},{ }^{1} \mathrm{H}^{\alpha}$, C' chemical shifts.

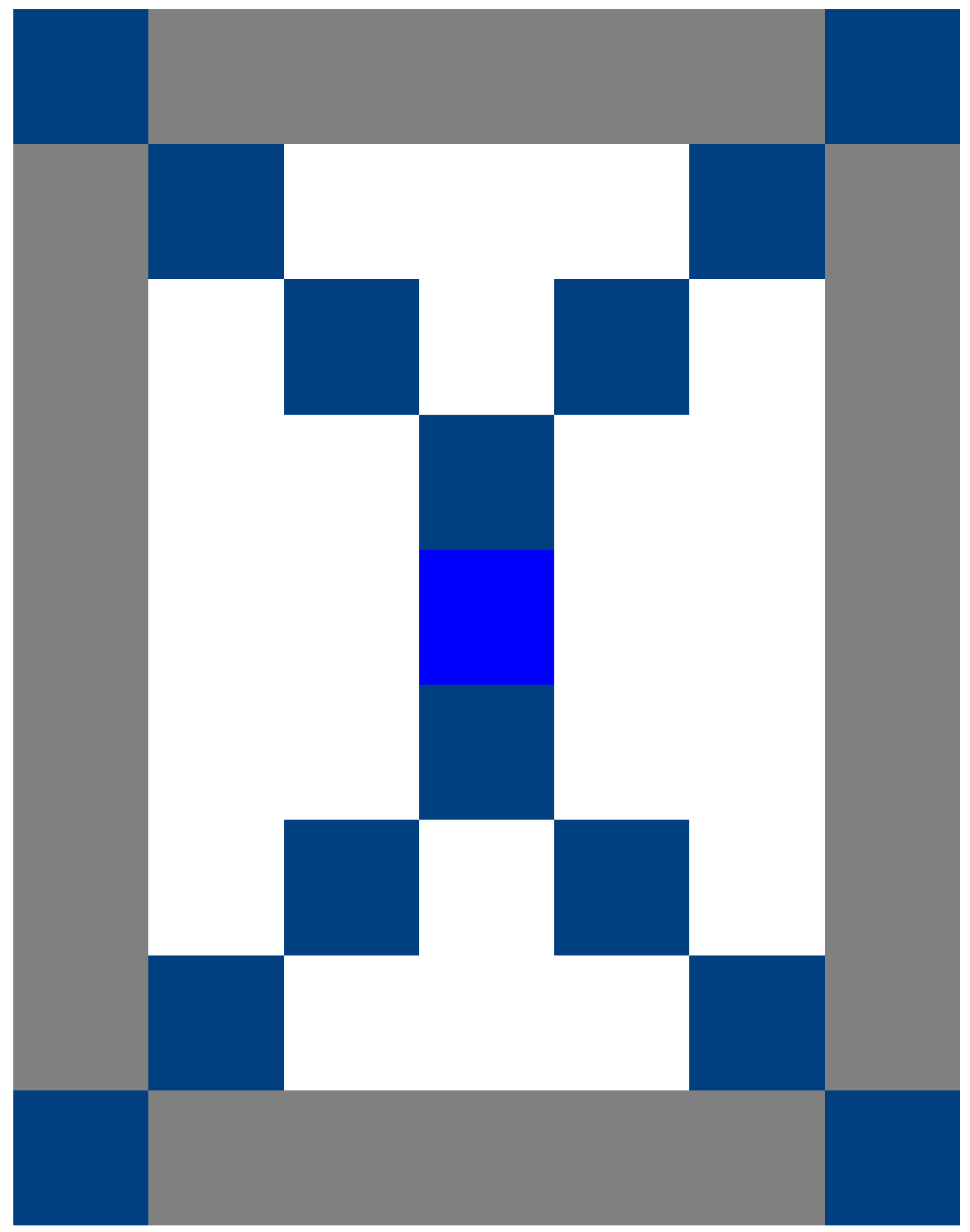




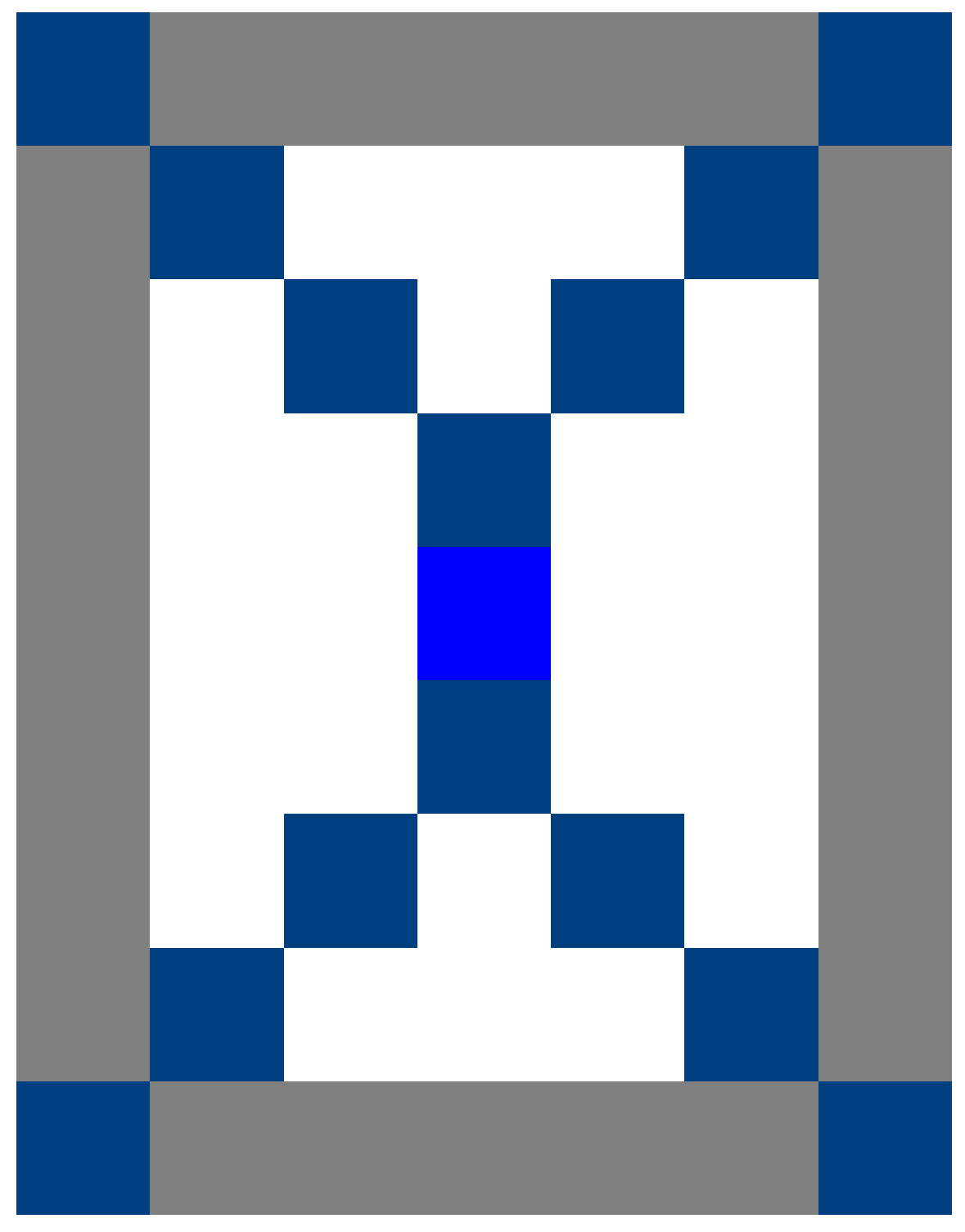




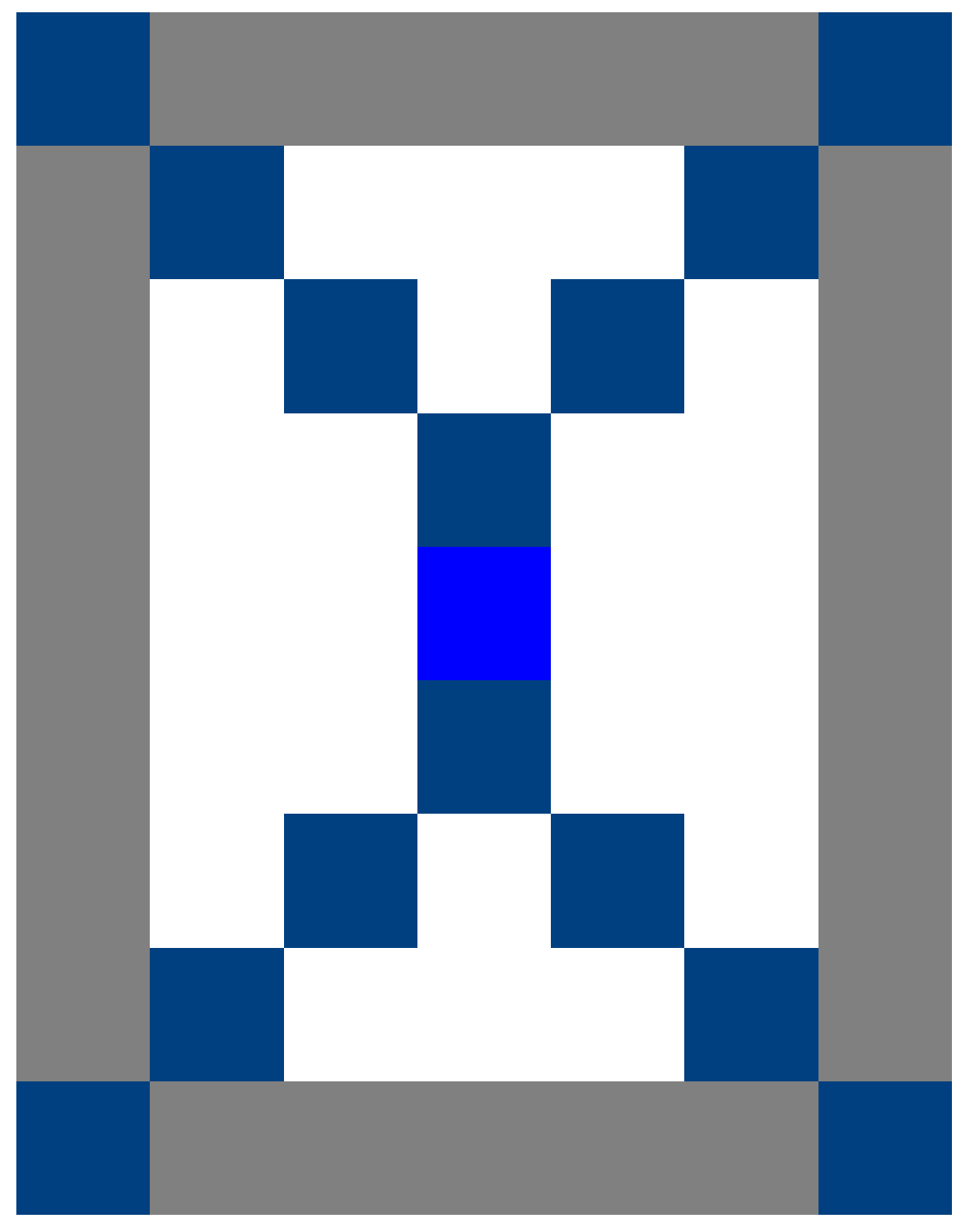




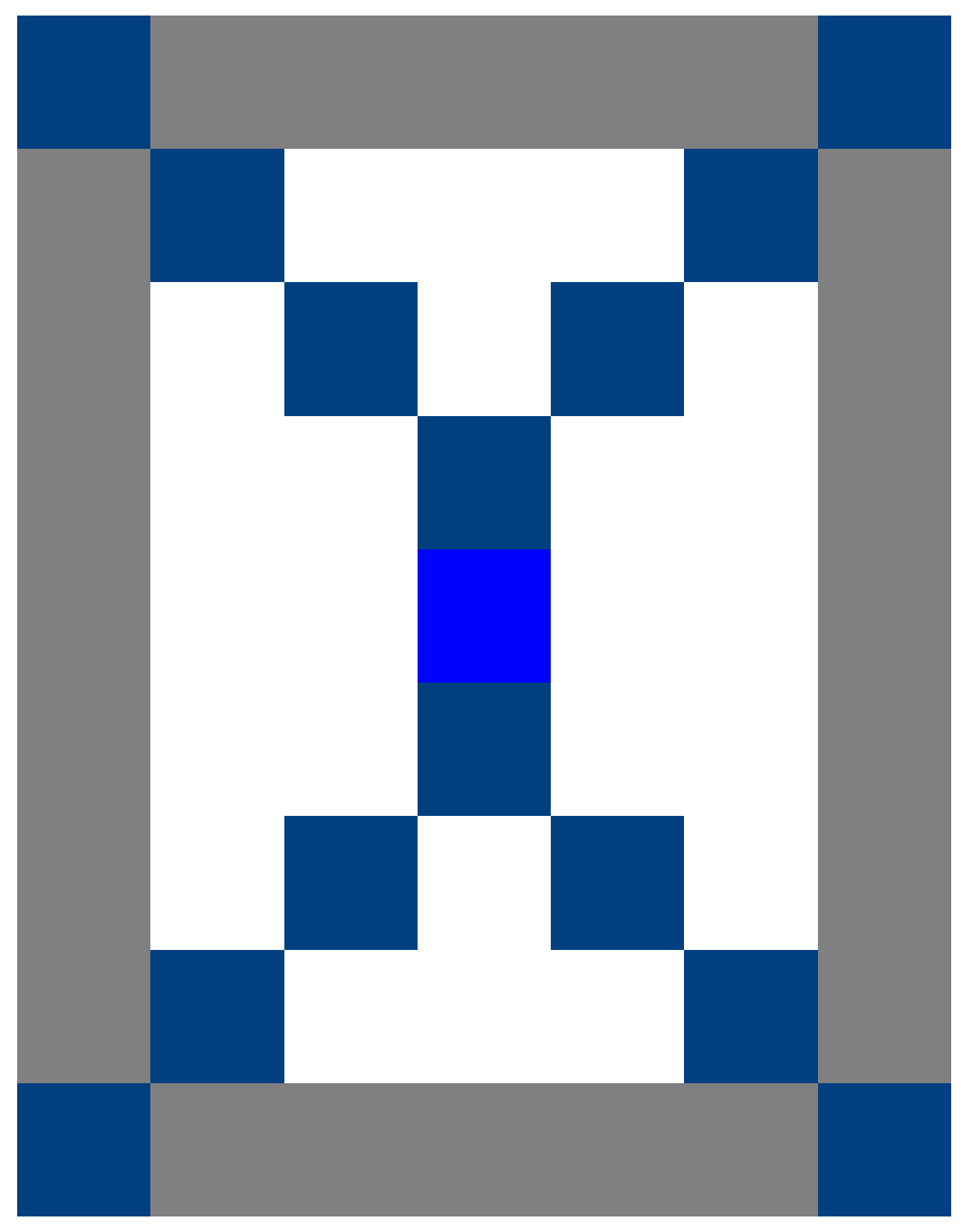

Fig. 3 : Characterization of the interaction of the IGFs with L- $h$ IGFBP2. Sensorgrams of (a) IG F-1, (b) IGF-2 immobilized on the surface of a CM5 chip with L- $h$ IGFBP2 as analyte. (c) Overlay of the $2 \mathrm{D}\left[{ }^{15} \mathrm{~N}-{ }^{1} \mathrm{H}\right]$ HSQC spectra of L- $h$ IGFBP2 (blue) and IGF-1: L- $h$ IGFBP2 (red). Inset shows an expanded view of the region where residues show maximum chemical shift perturbations (residues 150-170). (d) Combined $\left({ }^{15} \mathrm{~N}\right.$ and ${ }^{1} \mathrm{HN}$ ) chemical shift difference plot for L- $h$ IGFBP2 residues upon addition of IGF-1, calculated using . The dotted line is shown at one standard deviation of the chemical shift differences. (e) Reported proteolysis sites on L- $h$ IGFBP2. (f) Residues of the linker domain were analyzed for their predicted propensity to lie within MoRF motifs using the web-based program MoRFpred. A line between the peak centers of the red and blue signals for L152 and R156 residues shows the largest chemical shift deviations (in the insert, Fig. $3 \mathrm{c})$.

Fig. $4: \mathrm{R}_{2}, \mathrm{R}_{1 \rho}$, and het-NOE plots for L- $h$ IGFBP2 (blue) and IGF-1: L- $h$ IGFBP2 (red) measured at ${ }^{1} \mathrm{H}$ resonance frequency of $800 \mathrm{MHz}$. A-asterisks mark signals with overlap.

Fig. 5: Spectral density function values at $J(0), J\left(\omega_{\mathrm{N}}\right)$, and $J\left(0.87 \omega_{\mathrm{H}}\right)$ frequencies for L- $h$ IGFBP2 (left) and the L-hIGFBP2:IGF-1 (right) complex at a ${ }^{1} \mathrm{H}$ resonance frequency of $800 \mathrm{MHz}$. A-asterisks mark 
signals with overlap.

Fig. 6: The difference in $S^{2}$ values (calculated using Eq. 1) between the free and bound forms of L- $h$ IGFBP2 as estimated from $J(0)$ and $J\left(\omega_{\mathrm{N}}\right)$ calculated from $\mathrm{R}_{1 \rho}$.

Fig. 7: Predicted structure of full-length IGFBP2 from AlphaFold protein structure database where the disordered linker domain is represented in red, starting from A97 residue to C191 as marked, with N- and C-terminal domains of FL-IGFBP2 represented in cyan and blue respectively. The helix in the linker domain was predicted by Alpha Fold only (https://alphafold.ebi.ac.uk/) and consistent with the fact that K150-E161 is more ordered in IGFBP2 as we can see from Fig. S4

\section{Hosted file}

image7.emf available at https://authorea.com/users/447873/articles/546789-igf-dependentdynamic-modulation-of-a-protease-cleavage-site-in-the-intrinsically-disordered-linkerdomain-of-human-igfbp2

Figure 1 


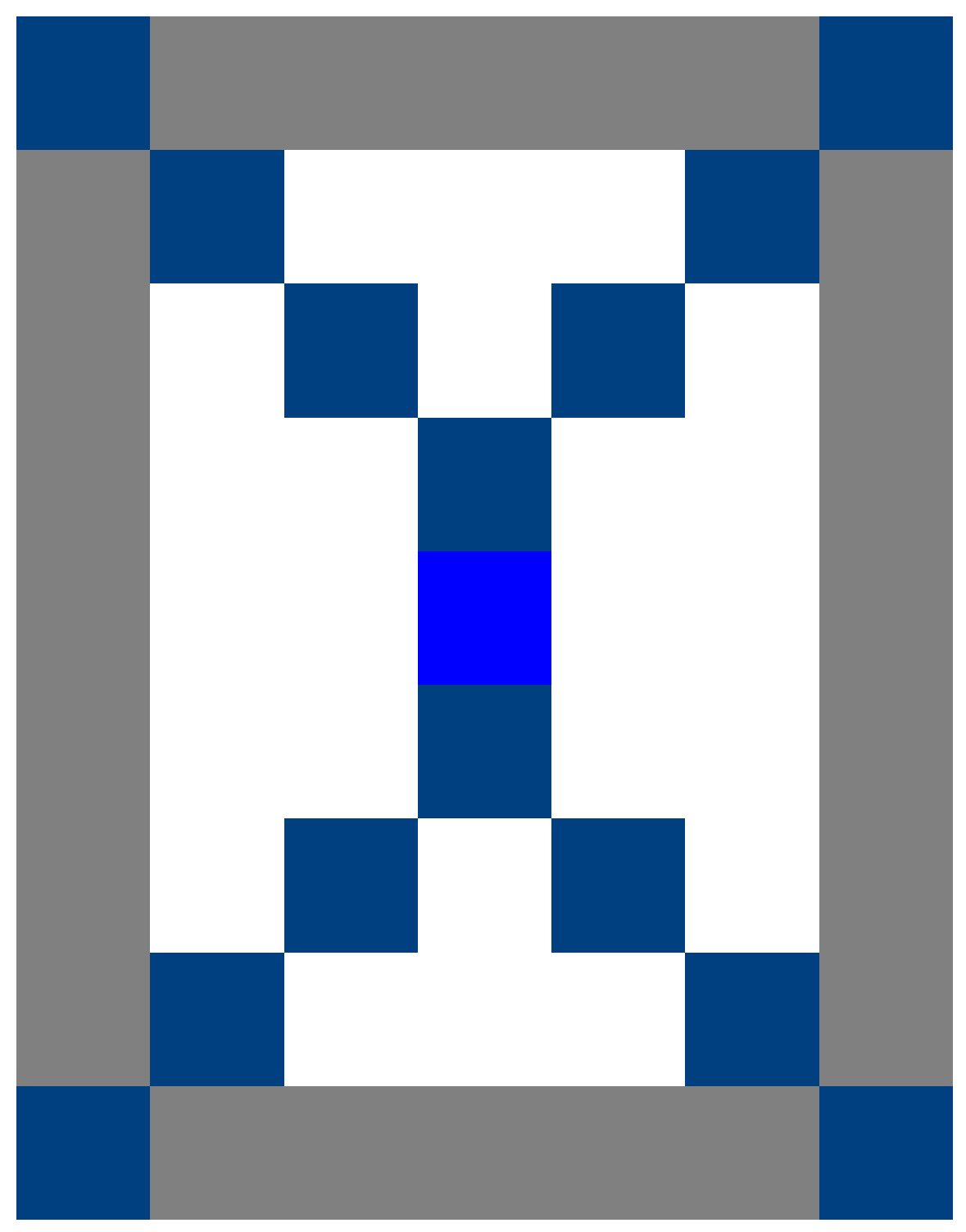

Figure 2 


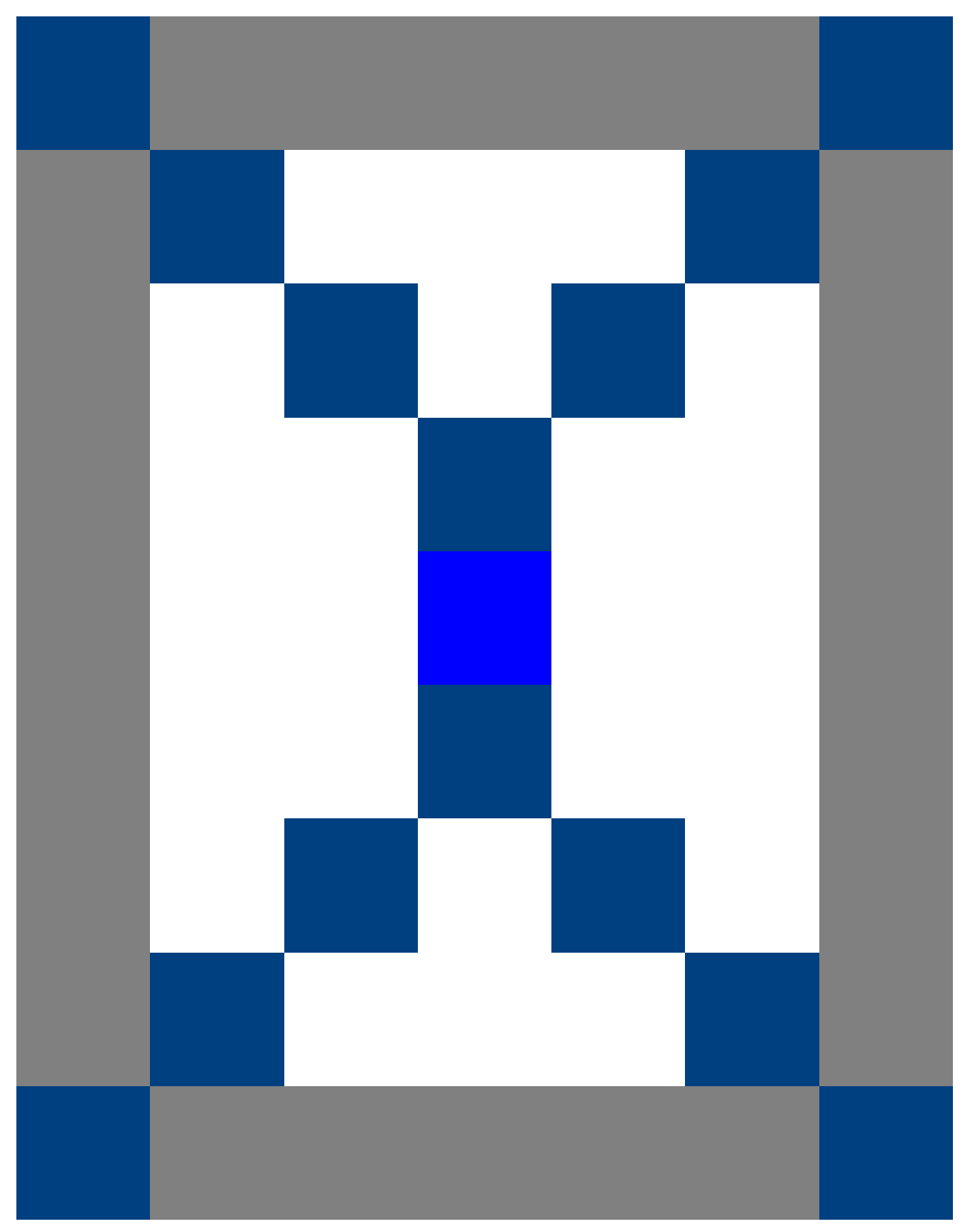

Figure 3 


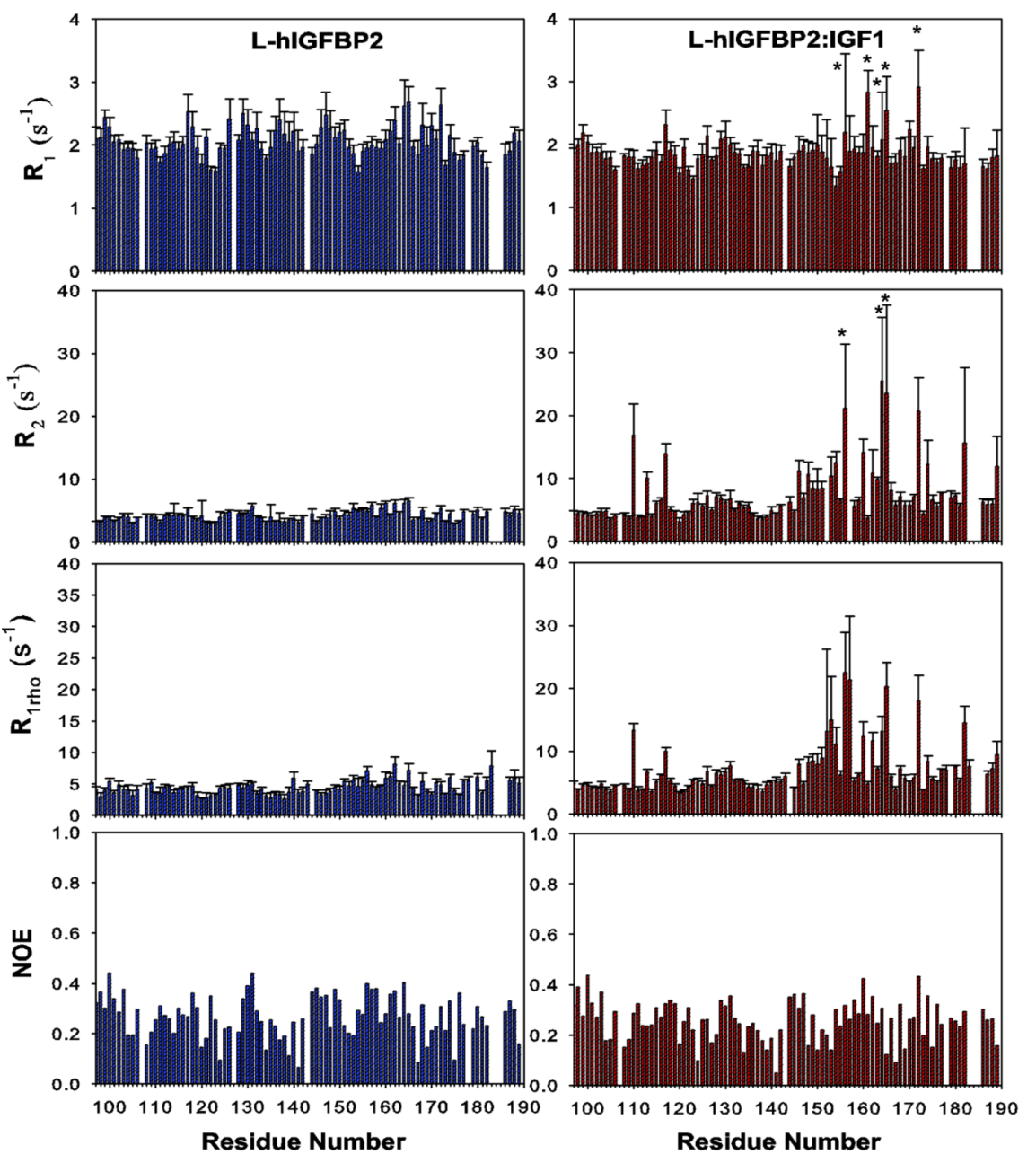

Figure 4 


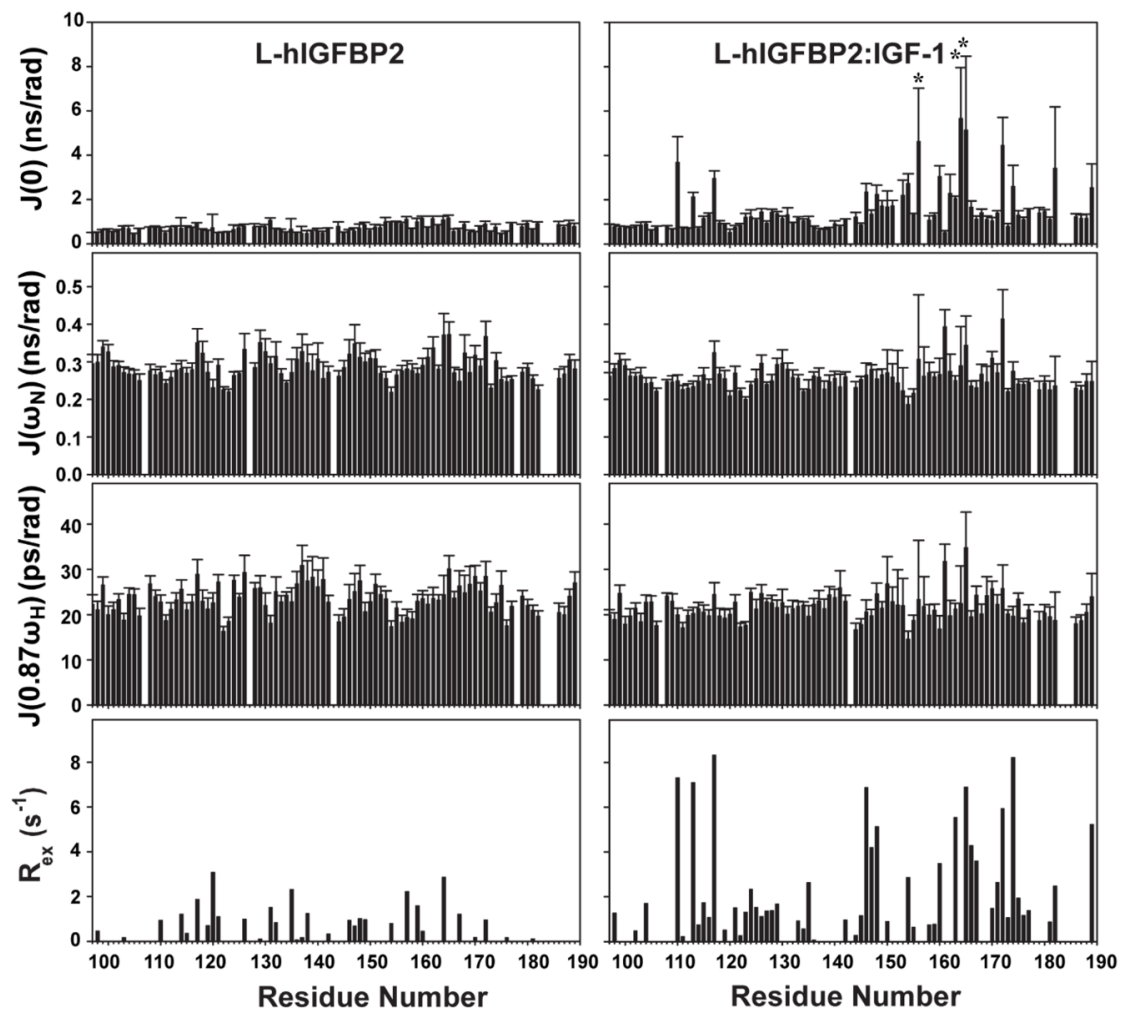

Figure 5

\section{Hosted file}

image12.emf available at https://authorea.com/users/447873/articles/546789-igf-dependentdynamic-modulation-of-a-protease-cleavage-site-in-the-intrinsically-disordered-linkerdomain-of-human-igfbp2

Figure 6 


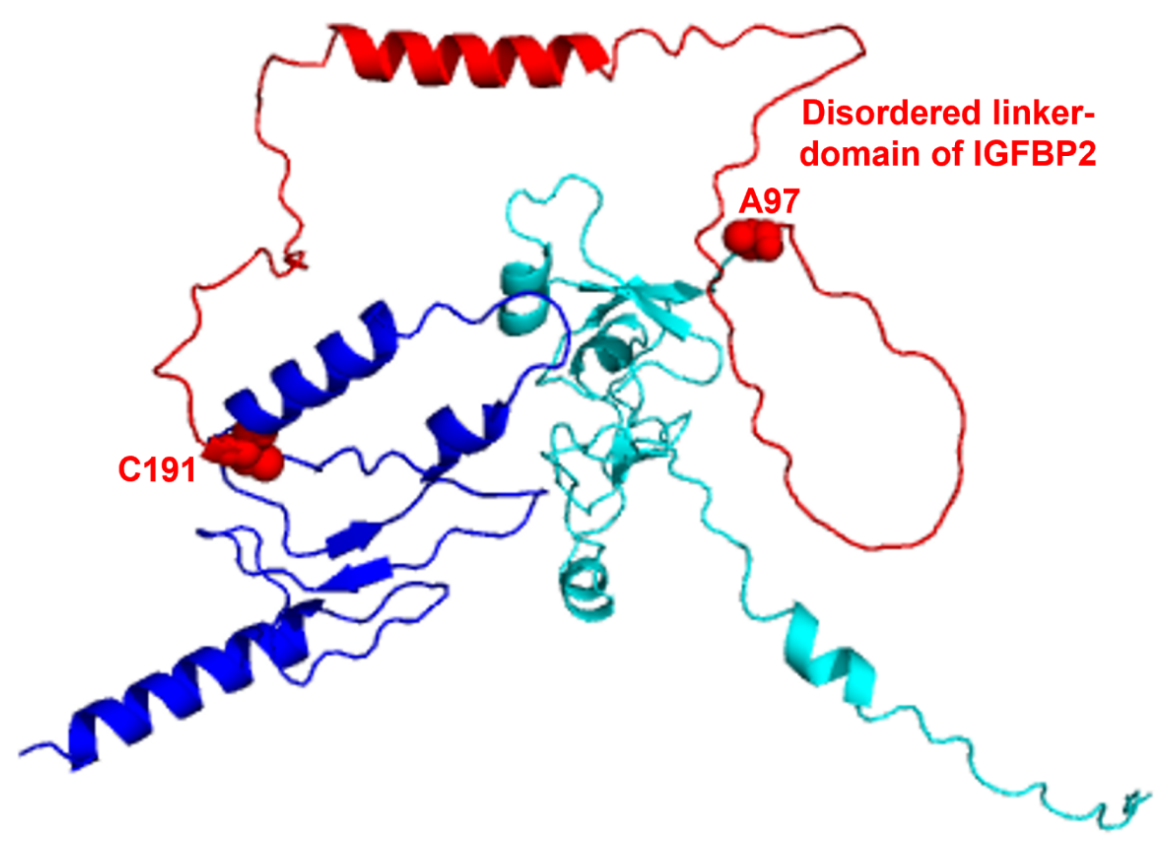

Figure 7 